\begin{tabular}{ccc}
\hline & International Journal of Engineering \&Technology, $7(3.12)(2018) 1090-1095$ \\
SPC & International Journal of Engineering \& Technology \\
Website www.sciencepubco.com/index.php/IJET & Research paper \\
\hline
\end{tabular}

\title{
HSV, Edge Preserved and Huffman Coding based Intra Frame High Efficient video Compression for Multimedia Communication
}

\author{
K. Sripal Reddy ${ }^{1}$, B. Leelaram Prakash ${ }^{2}$ \\ ${ }^{1}$ Research Scholar, Koneru Lakshmaiah Education Foundation, Green Fields, Vaddeswaram Guntur Dist., Andhra Pradesh \\ ${ }^{2}$ Professor, Dhanekula Institute of Engineering \& Technology, Ganguru, Vijayawada, Andhra Pradesh \\ *Corresponding Author Email: ${ }^{1}$ k.sripalreddy@gmail.com, ${ }^{2}$ prakashvignan4368@gmail.com
}

\begin{abstract}
High Efficiency Video Coding (HEVC) is another pressure standard for high resolution video content, which just needs $50 \%$ of the bit rate of H.264/Advanced Video Coding (AVC) at the same perceptual quality. Be that as it may, the computational intricacy is increments dramatically to adopt quad-tree organized Coding Unit (CU). In this document a new Hue Saturation Lightness (HSV), Edge Preserving and Huffman coding (HC) based intra frame high efficient video compression algorithm is introduced which is named as HSV-EPHCIFHEVC. To increase the compression ratio of the video frames Huffman and Differential Pulse Code Modulation (DPCM) encodings are used. To improve the de-compressed frame quality in compression Sharpening filter based Edge preserving technique is used. This HSV-EPHC-IFHEVC algorithm provides much better performance compared to existing systems. The performance measurement is in the terms of MSE, PSNR, RMSE and Execution time.
\end{abstract}

Keywords: High efficient video compression; Intra frame coding; Hue Saturation Lightness (HSV); Edge Preserving; Differential pulse-code modulation and Huffman coding.

\section{Introduction}

Over the most recent couple of years the request of the multimedia administrations i.e., the telecom, transmission and getting the video, sound and other information has expanded [1]. In the new generation Transmission of mixed media sharing is major challenge due to the very higher bandwidth (BW) requirement, the lesser delay is essential because of protocol stack between task and the aftereffect of blunder propagation inside the video succession in case of information parcel misfortunes. To conquer these constraints new video pressure standard Video Coding Experts Group (VCEG) is presented [2]. The prevailing standard for video coding is H.264 [3] and is utilized to convey an extensive variety of video correspondence offices.

Whenever, H.264 necessitates enormously high BW, constructing the transport of High-Definition (HD) video facilities unreasonable. Its successor, H.265 [4], is standardized by the Moving Picture Experts Group (MPEG) in 2012 and is expected to reduce the bit rate characterized to H.264 High Profile by about 50\% while maintaining comparable subjective quality [5]. Therefore HEVC is a more practical choice for delivering HD and Ultra HD (UHD) video content to consumers using in digital communication [6]. However, the coding efficiency still cannot meet real time requirements. A gradient based fast IM decision system was introduced [7] to decrease the computational difficulty of H.265, where the distribution of the gradient-mode histogram is engendered to achieve the intra prediction (IP) by using the lesser fragment of the candidate methods.
The firmness act of H.265 is associated with the numerous global standards [8], and parallel effects were reported in the JCT-VC study [9].

The greatest way to calculate the compression enactment is measures the subjective quality as seeming by genuine person viewers. The objective of the particular valuation is quantify [9]. The extent feasible, the bit rate savings for which like subjective quality is achieved for the H.265 test model compared to a similarly configured AVC encoder and decoder. The subjective assessments were conducted using nine test sequences with full $\mathrm{HD}$ 1920 X1080 and wide VGA (WVGA) 832X480 resolutions at four bit rate/quality points each. Cho et al. [10] utilized Bayes decision rule method for early termination of the coding unit. The statistical values of the previous frames can't improve the IP process performance. Zhang et al. [11] proposed a fast Intra Mode(IM) decision which based on the RD cost estimation.. C.U. Lee et al [12] proposed a method based on the depth level of CU. This can be done by consider the temporal correlation with neighboring Unit. Cus. Shen et al [13] presented a fast CU size decision algorithm according to texture homogeneity and coding information of neighboring CUs. However, when the texture information of the coding area is complex, the coding performance loss is relatively high for the prediction error of CU size selection. From the study of all the existing systems, it is found that they consist of some advantages and disadvantages. In this paper a new HSV, Edge Preserving and $\mathrm{HC}$ based Intra Frame High Efficient Video Compression algorithm is introduced (HSV-EPHC-IFHEVC). By using this HSV-EPHC-IFHEVC algorithm which provides much better performance compared to existing systems. 


\section{HSV-EPHC-IFHEVC System}

Block (BLK) based video coding is one of the mostly used compression technique for video. In this HSV-EPHC-IFHEVC system, Video frames are Edge preserved by the help of sharpening filter. The edge sharpened frame is split into coding BLK's. The BLK coding has been classified into two different types such as inter-frame coding technique and Intra-frame(IF) Coding . In this paper Intra-frame prediction coding technique is used for video compression. The block diagram of the HSV-EPHCIFHEVC system is shown in the Fig.1

Compression: From the block diagram HSV-EPHC-IFHEVC system it is seen that the input video is converted into frames, frame edges are preserved by the help of sharpening filter. The edge sharpened frames are split into coding BLK $\mathrm{s}$ (in the size of $16 \times 16,8 \times 8$ and $4 \times 4$ ). Each BLK is transformed by the $8 \times 8$ DCT. After the transformation to improve the frame clarity, the video Quantization (Qzn) and Zig-zag scanning is applied. To minimize the compressed bit size minimum values are encoded by the HC and maximum values are encoded by the Difference Pulse Code Modulation. Finally, Huffman encoded bits and DPCM encoded bits are cascaded.

Decompression: For decompression process, inverse process of compressing has been done. Finally the performance of the HSV-
EPHC-IFHEVC is measured. The algorithm of the HSV-EPHCIFHEVC system is shown below

\subsection{Algorithm of HSV-EPHC-IFHEVC System}

Step 1: Start frame separation.

Step 2: Initialize the Coding Block Tree $(\mathrm{CBT})$ and Prediction Unit(PU) blocks.

Step 3:Start Intra Prediction(IP). From this IP, 35 modes are detected and these modes are combined as 1 output which is given in to the BLK extraction.

Step 4: The blocks are again split as CBT and PU blocks. Then these blocks are given into the DCT, Quantization and Zig-zag scanning.

Step 5: The compressed array of the each blocks are obtained from Zig-zag scanning.

Step 6: The compressed array is again compressed by the Huffman and DPCM encoding. Finally, the outputs from the Huffman and DPCM are cascaded and the cascaded values are stored (i.e., encoded value of frame).

Step7: The decoding is performed by using the encoded Huffman and DPCM values. The decoding process of this video compression is nothing but the reversible process of encoding.

\section{Compression}

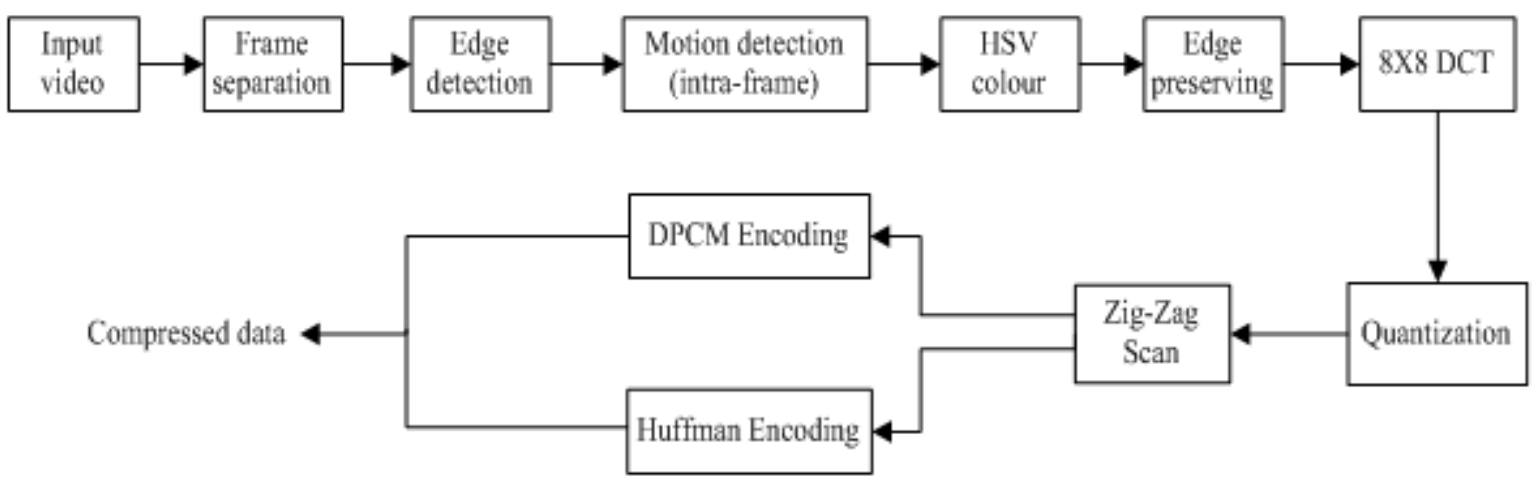

\section{Decompression}

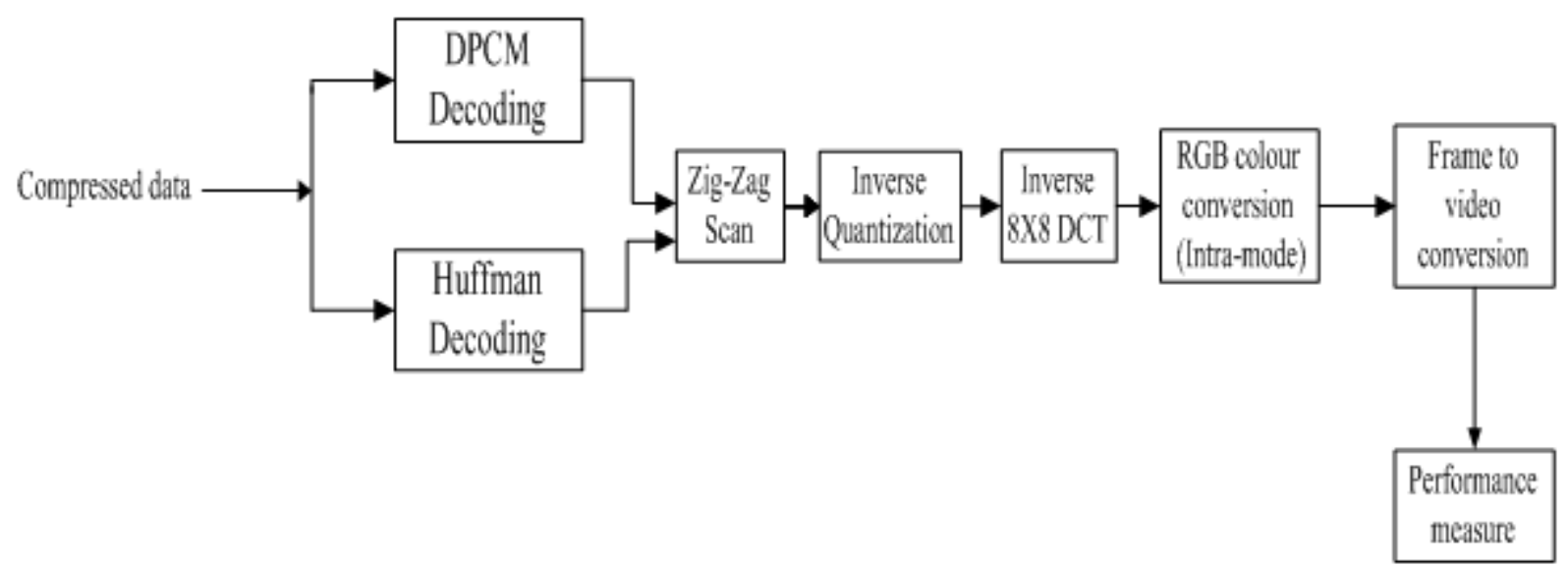

Fig. 1: Block diagram of the HSV-EPHC-IFHEVC system

\subsection{Intra Mode Predictions}

In MPEG-1 Standard(Std), I-Picture is coded by IF Coding. While using the I-Picture encoding, the spatial redundancy is reduced in the picture with comparing to other pictures. This Intra Mode(IM) coding procedure is same to the JPEG Std. So encoding I-Picture is lesser difficult compared to P-frame and B-frame. Decoding I-
Picture is differing procedure of encoding process (EP), so in this segment only EP is described. Earlier going advance, it should be known that the basic $\mathrm{CU}$ is a BLK which is an $8 \mathrm{X} 8$ matrix. And a macro-BLK consists of 6 blocks: 4 blocks of luminance (Y), one BLK of $\mathrm{Cb}$ chrominance, and one BLK of $\mathrm{Cr}$ chrominance. The macro-BLK building[14] is shows in the Fig. 2. 


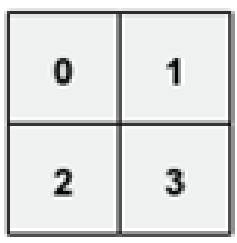

$\mathbf{Y}$
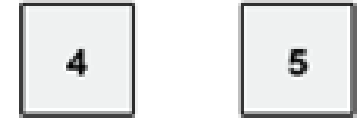

$\mathrm{Cb}$
Fig. 2: Macro BLK structure

The Intra Prediction(IP) prognosis is earlier used to decode the boundary models from spatially nearest blocks. In sequence to forecast a new prognosis BLK is PB. The initial image of a video various improvements have been familiarized in H.265 in the IP module because of the greater size of pictures, the collection of maintained coding BLK size (BS) has been greater than before. A Planer mode (PM) considers the continuity at BLK edges is preferred. using this the directionality is improved. The Intra Mode(IM) coding is an effective method to transmit the data for each BLK based on the different directional modes. H.265 funds a huge variety of BS, so it requires consistency across all BS. The initial image at each clear unsystematic access point in a video coded [15]. The Table-1 show the various prediction, IP modes with their corresponding index.

Table 1: IP mode

\begin{tabular}{|c|l|}
\hline IP Mode & IP Mode index \\
\hline PC Mode & M 0 \\
\hline AM & M 1 \\
\hline & M 2 to 34 \\
\hline
\end{tabular}

\subsubsection{Angular Prediction}

The angular prediction progression is functioning of spatial domain. There are 33 different directions are supported in Angular Mode(AM) of HEVC and these AM's are indexed from 2 to 34 in intra coding. The directions from AM is covered the angles of images related to horizontal, near-diagonal and near-vertical. A displacement parameter of an each is denoted as d, where the measure of $\mathrm{d}$ demonstrates the numeric part that is the pixel's removal communicated in $1 / 32$ pixel divisions of exactness, The $\mathrm{V}$ and $\mathrm{H}$ are indicated the vertical and level directionalities respectively [16]. The modes ten and twenty six are known as unadulterated $\mathrm{H}$ forecast and unadulterated vertical expectation individually. The AM extrapolates the fragments from the reference models are derived from mode 10 to mode 26 . This prediction achieves less complexity because of its directional value. The fragments located (FL) in a row are represented as extra FL When the direction selected is among 2 and 17,and it is located in the left Column which is extended into left innuendo column. The FL which are at the left column are concentrated as FL in the above row, when the direction selected is among 18 and 34 , is and it is extended into the best insinuation push. In the two cases the sections are concentrated and it has negative records. Once the insinuation section are known, each piece is anticipated by demonstrating its situation into the innuen-do parts and applying the favored expectation course. Fragment prediction equation for vertical modes i.e., AM's 18-34

$$
\begin{aligned}
& \left.P_{m, n}=\left(\left(32-w_{n}\right) * R_{i, 0}+w_{n} * R_{i+1,0}+16\right)\right\rangle>5 \\
& \left.c_{n}=(n * d)\right\rangle>5 \\
& w_{n}=(n * d) \& 31 \\
& i=m+c_{n}
\end{aligned}
$$

Fragment prediction equation for horizontal modes i.e., AM's 217:

$$
\begin{aligned}
& \left.P_{m, n}=\left(\left(32-w_{m}\right) * R_{i, 0}+w_{m} * R_{i+1,0}+16\right)\right\rangle>5 \\
& \left.c_{m}=(m * d)\right\rangle>5 \\
& w_{m}=(m * d) \& 31 \\
& i=n+c_{m}
\end{aligned}
$$

In this equations ((1) and (2)), $\mathrm{R}_{\mathrm{i}, \mathrm{j}}$ denotes the innuendo fragment and $\mathrm{P}_{\mathrm{m}, \mathrm{n}}$ denotes the fragment to be predicted, whereas $\mathrm{m}, \mathrm{n}$ are the spatial coordinates. The sub pixel location is between $R_{i, 0}$ and $\mathrm{R}_{\mathrm{i}+1,0} \mathrm{c}_{\mathrm{m}}$ and $\mathrm{c}_{\mathrm{n}}$ denoted pixel parameters corresponding to $\mathrm{m}$ and $\mathrm{n}$ coordinates.

$\mathrm{w}_{\mathrm{n}}$ denotes the parameter related to the weighed prediction, ' $\mathrm{i}$ ' indicates the innuendo fragments index, ' $>$ ' named a bit shift operation to the right and \& represents AND logical operation. The limits $c_{n}$ and $w_{n}$ be subject to manage in concentrated mode and $c_{m}$ and $w_{n}$ depend only on $\mathrm{m}$ manage for $\mathrm{H}$ approaches [17].

\subsubsection{Planer Mode}

The planer mode is similar to the H.264/MPEG-4 AVC, it is called as a mode 0 . The PM algorithm is recommended to the discontinuities along the structure of 2-D signal borderline. The continuities along the BLK edges is preserved by HEVC. By assuming surface with a smooth gradient, the number of PB fragment is calculated. These gradient are derived from the borderline fragment of neighboring blocks.

Fragment prediction equation for PM

$$
\begin{aligned}
& P_{x, y}^{V}=(N-y) * R_{x, 0}+y * R_{0, N+1} \\
& P_{x, y}^{H}=(N-x) * R_{0, y}+x * R_{N+1,0} \\
& \left.\left.P_{x, y}=\left(P_{x, y}^{V}+P_{x, y}^{H}+N\right)\right\rangle\right\rangle\left(\log _{2}(N)+1\right)
\end{aligned}
$$

In this eqn.(3), $\mathrm{x}$ and $\mathrm{y}$ named location of fragment. $\mathrm{N}$ stands for total amount of fragment. $\mathrm{V}$ and $\mathrm{H}$ are for vertical and horizontal respectively. $\mathrm{R}$ stands for reference fragment.

\subsubsection{Mode}

The DC mode is also identical to the AVC DC mode and it is named as a mode 1 . In larger frequency factors, this DC mode provides coarse prediction Incase of finely textured areas it is not effective. An authority fragments average is equal to the each fragment of the PB

\subsection{Discrete Cosine Transform (DCT)}

In MPEG-1 $8 * 8$ DCT helps to alter at $8 * 8$ pel BLK to another $8 * 8$ BLK. the energy value of the image is concentrated on topleft corner.

$$
\begin{aligned}
& \left.F(u, v)=1 / 4 C(u) C(v) \sum_{x=0}^{\tau} \sum_{y=0}^{\tau} f(x, y) \cos ((2 x+1) u \pi / 16) \cos (2 y+1) v \pi / 16\right) \\
& u, v, x, y=0,1,2 \ldots 7 \\
& c(j)=\frac{1}{\sqrt{2}}, j=0 \\
& c(j)=1, j>0
\end{aligned}
$$

Inverse two-dimension $8 \times 8$ DCT transform

$$
F(x, y)=1 / 4 \sum_{u=0}^{\tau} \sum_{v=0}^{\tau} C(u), C(v) F(u, v) \cos ((2 x+1) u \pi / 16) \cos ((2 y+1) v \pi / 16)
$$




\subsection{Quantization}

Quantization(Qzn) is to reduce the range of amounts to a single small value, so that we can use less no. of bits to represent a large amount. For example, we can round a real to an integer. In MPEG-1, use a matrix called quantizer $(\mathrm{Q}[\mathrm{m}, \mathrm{n}])$ to define $\mathrm{Qzn}$ step. Each and Every time when a pels matrix $(X[m, n])$ with the same size $\mathrm{Q}[\mathrm{m}, \mathrm{n}]$ comes, use $\mathrm{Q}[\mathrm{m}, \mathrm{n}]$ to divide $\mathrm{X}[\mathrm{m}, \mathrm{n}]$ to get Qzned matrix $\mathrm{X}_{\mathrm{q}}[\mathrm{m}, \mathrm{n}]$.

Quantization Eq.:

$$
X_{q}[m, n]=\operatorname{Round}(X[m, n] / Q[m, n])
$$

Inverse Quantization Eq:

$$
X^{\prime}[m, n]=X_{q}[m, n] * Q[m, n]
$$

Inverse Qzn is to reconstruct original value. But we can see Qzn eqn. uses Round ( ) function to get a nearest integer value, so that remodeled value will not be the same as primary value. The characteristics among primary value and remodeled value from Qzned value is also named the Qzn error. In general if we carefully design $\mathrm{Q}[\mathrm{m}, \mathrm{n}]$, visual quality will not be damaged.

\subsection{Zig-zag scan}

Quantization afterwards most AC attitudes are in the form of zero, by using zigzag scan which can gather even more consecutive zeros.

\subsection{Huffman Coding}

Huffman Coding (HC) is recommend in 1952 by Huffman. HC is the lossless data compression technique and minimum redundancy method. The HC idea is to assign variable range codes to input characters, lengths of the assigned codes are based on the frequencies of corresponding characters. Huffman's greedy technique looks at the occurrence of each character and it as a binary string in an optimal way. The method accomplishes its aim by allowing character to vary in range. Shorter codes are assigned to the most intermittently used character, and protracted codes to the symbols which appear less intermittently in the string. Code word range are no protracted fixed like ASCII. Code word range vary and will be shorter for the more intermittently used characters [15].

\subsection{Differential Pulse-Code Modulation}

DPCM is an encoding technique, which transfers an analog data to a digital data. The analog data is tested and then the characteristics between the actual model value and its predicted value is quantized. A predicted value of the actual model is based on the value of the previous model or the values of the previous models [16]. DPCM is also known as Digital pulse modulation, its working in the Prediction algorithm.

\section{Results and Discussion}

The HSV-EPHC-IFHEVC method was successfully implemented by using the online available data sets. The HSV-EPHC-IFHEVC system was analyzed with the help of MATLAB stimulator software version 2018a. The entire work is done by using I7 system with 8 GB RAM. The performance of the HSV-EPHC-IFHEVC method was measured by the terms of PSNR, RMSE, MSE and Elapsed time. In this HSV-EPHC-IFHEVC method process is explained with ant_maze.avi video. The ant_maze.avi video features are given in the Table- 2 .
Table 2: ant maze.avi video features

\begin{tabular}{|c|c|}
\hline Duration & $3.366 \mathrm{sec}$ \\
\hline Bits per pixel & 24 \\
\hline Frame rate & 30 \\
Height & 340 \\
\hline Width & 480 \\
\hline Video format & RGB24 \\
\hline Number of frames & 101 \\
\hline
\end{tabular}

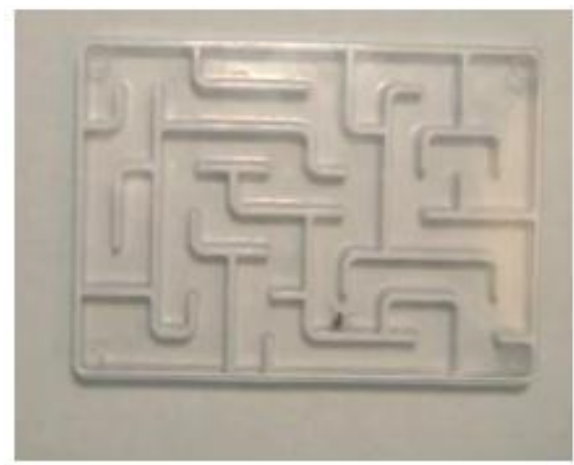

Fig. 3: ant_maze video framer

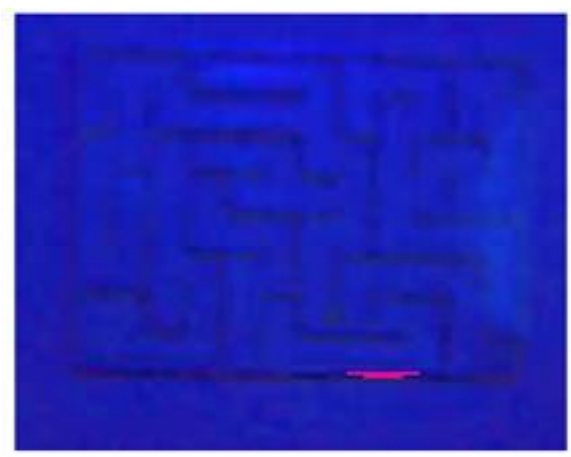

Fig. 4: ant_maze video HSV converted frame

The input video frames are separated and are shown in Fig.3. The separated video frame is converted into the Hue Saturation Lightness (HSV) format which is shown in Fig.4. HSV frame planes are separated, each plane is separately processed.

The plane separated video frame is edge preserved by the sharpening filter, which improves the quality of frame at the time of image decompression. The edge preserved sharpening filter frame is shown in the Fig.5.

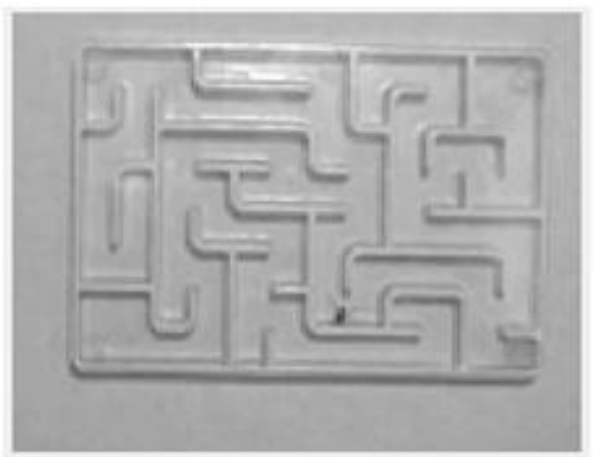

Fig. 5: Edge preserved sharpening filter frame

Edge preserved filter sharpens the frame BLK which is separated as shown in Fig.5 encodes by the help of inter and intra prediction. The index positions of all blocks are stored. Encoded blocks are transformed by the DCT. DCT transferred blocks are normalized by the Qzn matrix. Normalized matrix is scanned by the zigzag 
scan, which provides the array output for that block. All blocks are processed and it is saved as an array. From the block, array's are collected and cascaded for Encoding Process. The cascaded array maximum values are collected and encoded by the DPCM Encoding. Cascaded array minimum values are collected and are encoded by the Huffman coding. Finally both the DPCM Encoded data and Huffman encoded data are cascaded which is compressed data.

In the decompression, compressed data is considered as an input data which is separated into two arrays such as maximum value array and minimum value array. Minimum value array is given to the Huffman decoding and maximum value array is given to the DPCM decoding which is decoded by the respective technique. After that decoded values are cascaded. That cascaded array is given to the inverse zig-zag scan which provides matrix output. Inverse Qzn and inverse DCT are then applied. By using this inter and intra, encoded image is reconstructed with the help of index values. Finally the performance is measured for all frames in terms of PSNR, MSE, RMSE and execution time. For the performance evaluation some other Videos are also considered. The features for those videos are shown in the Table 3.

Table 3: Different input video features

\begin{tabular}{|c|c|c|c|c|}
\hline File name (.avi) & Walk1 & Walk2 & VIP Warn signs & VIP mosaicking \\
\hline Duration (s) & 2.0667 & 2.9200 & 9.9000 & 24 \\
Bits per pixel & 24 & 24 & 24 & 24 \\
Frame rate & 15 & 25 & 30 & 15 \\
\hline Height & 260 & 180 & 360 & 240 \\
Width & 260 & 144 & 180 & 320 \\
Video format & RGB24 & RGB24 & RGB24 & RGB24 \\
\hline Number of frames & 31 & 73 & 297 & 71 \\
\hline
\end{tabular}

\subsection{Mean Square Error}

The definition of an MSE differs according to whether one is describing an estimator or a predictor. The mathematical representation of Image MSE is shown in Eq 8.

$$
M S E=\frac{1}{P Q} \sum_{m=0}^{P-1} \sum_{n=0}^{Q-1}[I(m, n)-J(m, n)]^{2}
$$

Where ' $\mathrm{I}$ ' is the original frame and ' $\mathrm{J}$ ' is the decompressed frame

\subsection{RMSE}

The RMSE is the standard deviation of the residuals prediction error. The mathematical representation of RMSE is given in Eq. 9

$$
R M S E=\sqrt{M S E}
$$

\subsection{PSNR}

PSNR, is used to find the quality of image based on the difference between pixels of two images. It is used to define the quality of rebuilt picture compared with original picture. PSNR is defined as given in Eq.10

$$
P S N R=10 \log _{10}\left(\frac{\operatorname{Max}_{I}^{2}}{M S E}\right)
$$

Table 4: Different videos for HSV-EPHC-IFHEVC system performance evaluation

\begin{tabular}{|c|c|c|c|c|}
\hline File name (.avi) & Walk1 & Walk2 & Vipwarnsigns & vipmosaicking \\
\hline \multirow{2}{*}{ Sample frame } & & & & \\
& & & & \\
\hline PSNR (dB) & 27.67163 & 40.26690 & 35.87158 & 36.95420 \\
\hline MSE & 110.12487 & 61.65320 & 169.72192 & 133.07718 \\
RMSE & 10.33801 & 7.8512 & 13.0246 & 11.5154 \\
Elapsed time (s) & 350.9787 & 191.0416 & 333.518 & 370.833 \\
\hline
\end{tabular}

Table 5: Performance evaluations of different videos using existing systems

\begin{tabular}{|c|c|c|c|c|}
\hline File name (.avi) & Walk1 & Walk2 & Vipwarnsigns & vipmosaicking \\
\hline PSNR (dB) & 23.9470 & 19.978 & 20.1669 & 18.274 \\
\hline MSE & 126.41 & 165.85 & 150.02 & 101.088 \\
\hline RMSE & 11.22 & 12.87 & 12.24 & 10.05 \\
\hline Elapsed time (s) & 430.422 & 341.20 & 354.445 & 383.117 \\
\hline
\end{tabular}

The performance of the HSV-EPHC-IFHEVC system for different system [21] and HSV-EPHC-IFHEVC system is shown in the input videos is shown in the Table-4. The HSV-EPHC-IFHEVC system performance is also compared with the existing system [21] which is implemented and tested. The performance evaluaFig.7. Compared to the existing system the HSV-EPHC-IFHEVC system requires less Elapsed time.

The PSNR comparison of the existing system [21] and HSVEPHC-IFHEVC system is shown in the Fig.6. Compared to the existing system, HSV-EPHC-IFHEVC system provides much better PSNR rate. The Elapsed time comparison of the existing 


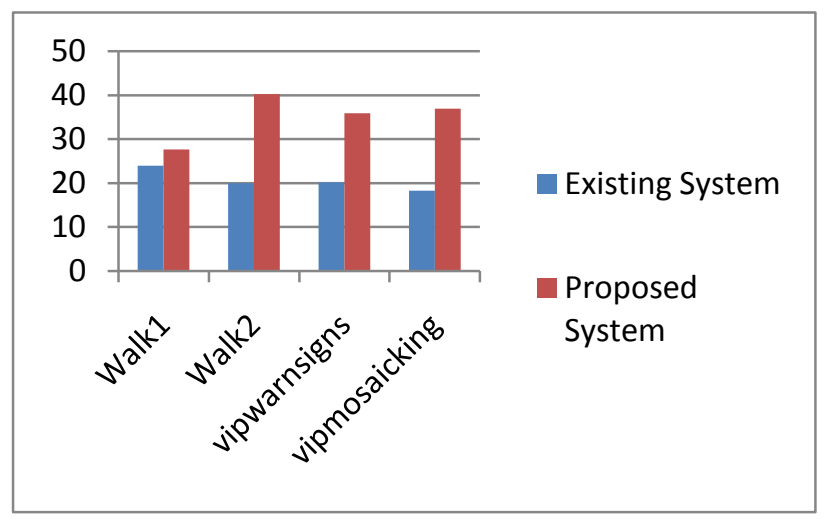

Fig. 6: PSNR comparison of the existing system and HSV-EPHCIFHEVC

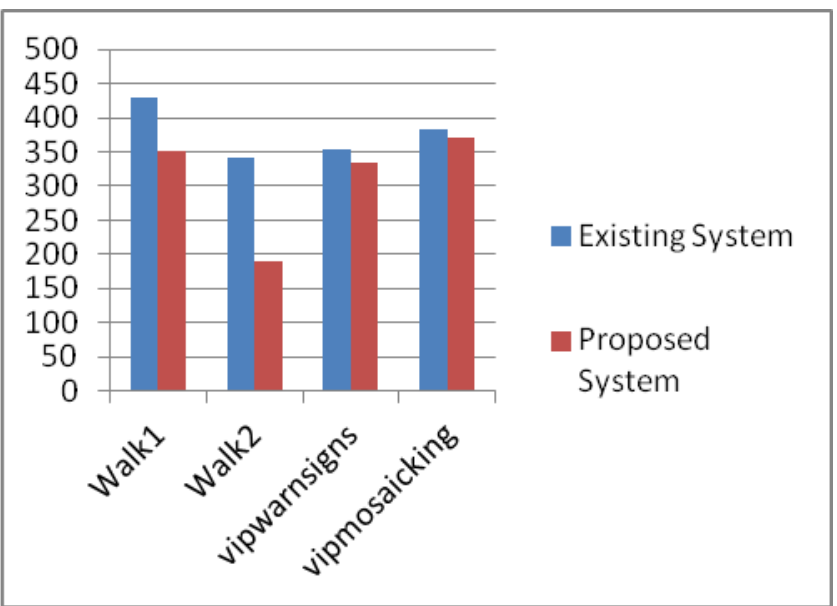

Fig. 7: Elapsed time comparison of the existing system and HSV-EPHC IFHEVC

\section{Conclusion}

In this document a novel Huffman and DCPM encoding based intra frame HSV-EPHC-IFHEVC technique is introduced for High efficient video compression. To improve the decompressed frame quality, compression sharpening filter based Edge preserving technique is used. The experimental results show that the HSV-

EPHC-IFHEVC technique provides better presentation in the terms of PSNR, and Implementation time compared to the existing system. In the future work some optimization techniques may be used to minimize the execution time.

\section{References}

[1] Bilgin, Ali, and Michael W. Marcellin. "JPEG2000 for digital cinema." In Circuits and Systems, 2006. ISCAS 2006. Proceedings. 2006 IEEE International Symposium on, pp. 4-pp. IEEE, 2006.

[2] Sullivan, Gary J., Jens Ohm, Woo-Jin Han, and Thomas Wiegand "Overview of the high efficiency video coding (HEVC) standard." IEEE Transactions on circuits and systems for video technology 22, no. 12 (2012): 1649-1668

[3] Chen, Ying, Miska M. Hannuksela, Teruhiko Suzuki, and Shinobu Hattori. "Overview of the MVC+ D 3D video coding standard." Journal of Visual Communication and Image Representation 25, no. 4 (2014): 679-688

[4] Nightingale, James, Qi Wang, Jose M. Alcaraz Calero, Ian Owens, and Christos Grecos. "Enhancing visual communications capabilities in tactical networks." In Military Communications and Information Systems (ICMCIS), 2015 International Conference on, pp. 1-9. IEEE, 2015

[5] Wien, Mathias. High efficiency video coding: Coding tools and specification. Springer, 2014.
[6] Fang, Fang. "Energy efficient resource allocation for NonOrthogonal Multiple Access (NOMA) systems." PhD diss., University of British Columbia, 2017.

[7] Jiang, Wei, Hanjie Ma, and Yaowu Chen. "Gradient based fast mode decision algorithm for intra prediction in HEVC." In Consumer Electronics, Communications and Networks (CECNet), 2012 2nd International Conference on, pp. 1836-1840. IEEE, 2012.

[8] Ohm, J-R., Gary J. Sullivan, Heiko Schwarz, Thiow Keng Tan, and Thomas Wiegand. "Comparison of the coding efficiency of video coding standards - including high efficiency video coding (HEVC)." IEEE Transactions on circuits and systems for video technology 22, no. 12 (2012): 1669-1684.

[9] Baroncini, V., G. J. Sullivan, and J. R. Ohm. "Report on Preliminary Subjective Testing of HEVC Compression Capability, document JCTVCH1004." Joint Collaborative Team on Video Coding (JCT-VC) of ITU-T SG 16 (2012).

[10] S. Cho and M. Kim, Fast CU splitting and pruning for sub-optimal CU partitioning in HEVC intra coding, IEEE Transactions on Circuits and Systems for Video Technology, vol. 23, No. 9, pp. 15551564, Sep. 2013.

[11] Hao Zhang; Zhan Ma, "Fast Intra Mode Decision for High Efficiency Video Coding (HEVC)," IEEE Transactions on Circuits and Systems for Video Technology, vol. 24, no. 4, pp. 660668, April 2014

[12] H. S. Lee, K.Y. Kim, and G. H. Park, "Fast encoding algorithm based on depth of coding unit for high efficiency video coding," Journal of optical engineering, vol. 51, no. 6, pp. 067402, June, 2012.

[13] Liquan Shen, Zhaoyang Zhang, Zhi Liu, "Effective CU Size Decision for HEVC Intra coding," IEEE Transactions on Image Processing, vol.23, no.10, pp.4232-4241, Oct. 2014.

[14] Patel, Dhruti B., Divya M. Chauhan, and Ketki C. Pathak. "Edges based intra-prediction in High Efficiency Video Coding (HEVC) standard." In Engineering and Technology (ICETECH), 2016 IEEE International Conference on, pp. 995-1000. IEEE, 2016.

[15] Sullivan, Gary J., Jens Ohm, Woo-Jin Han, and Thomas Wiegand. "Overview of the high efficiency video coding (HEVC) standard." IEEE Transactions on circuits and systems for video technology 22, no. 12 (2012): 1649-1668.

[16] Lainema, Jani, and Kemal Ugur. "Angular intra prediction in high efficiency video coding (HEVC)." In Multimedia Signal Processing (MMSP), 2011 IEEE 13th International Workshop on, pp. 1-5. IEEE, 2011

[17] Philipp Helle, Simon Oudin, Benjamin Bross, Detlev Marpe, M. Oguz Bici, Kemal Ugur, Joel Jung, Gordon Clare, and Thomas Wiegand, December 2012. Block Merging for Quad tree-Based Partitioning in HEVC. IEEE Transactions on Circuits and System for Video Technology.

[18] Lin, Yu-Kun, and Tian-Sheuan Chang. "Fast block type decision algorithm for intra prediction in H. 264 FRext." IEEE Internationa Conference on Image Processing ICIP 2005, vol. 1, pp. I-585.

[19] Rachmawati, D., A. Amalia, and J. Surya. "Combination of Huffman Coding Compression Algorithm and Least Significant Bit Method for Image Hiding." In Journal of Physics: Conference Series, vol. 801, no. 1, p. 012059. IOP Publishing, 2017.

[20] Tomar, Rime Raj Singh, and Kapil Jain. "Lossless Image Compression using Differential Pulse Code Modulation and Its purpose." International Journal of Signal Processing, Image Processing and Pattern Recognition 8, no. 9 (2015): 249-256.

[21] Kurihara, Shin, Suguru Hirokawa, and Hisakazu Kikuchi. "A New Scheme of Distributed Video Coding Based on Compressive Sensing and Intra-Predictive Coding." IEICE TRANSACTIONS on Information and Systems 100, no. 9 (2017): 1944-1952. 